\title{
RIPENSARE IL FUTURO DELL'ACQUA SUL NOSTRO PIANETA
}

\section{RECENSIONE ARTICOLO}

DIAS, Deusira Nunes Di Lauro ${ }^{1}$

DIAS, Adailton Di Lauro ${ }^{2}$

DIAS, Deusira Nunes Di Lauro. DIAS, Adailton Di Lauro. Ripensare il futuro dell'acqua sul nostro pianeta. Revista Científica Multidisciplinar Núcleo do Conhecimento. anno 04, Ed. 08, Vol. 06, pp. 19-32. nell'agosto 2019. ISSN: 24480959

\section{RIEPILOGO}

Considerato il liquido più importante, prezioso e indispensabile per la sopravvivenza della vita umana sulla Terra, l'acqua è stata motivo di costante preoccupazione, perché il modo in cui la società si è appropriata e ha utilizzato le risorse idriche sul pianeta ha Impegnati per la qualità, così come l'accesso di molte comunità a questa risorsa vitale per il mantenimento della vita. Questo lavoro intende analizzare e discutere strategie che garantiscano la conservazione e l'uso sostenibile dell'acqua sul pianeta, affrontando che, in alcune regioni del Brasile e del mondo, sono già con estrema scarsità d'acqua e, secondo alcuni autori, questo problema tende ad aumentare sempre di più a causa di diversi fattori, e i principali sono la rapida crescita della popolazione e la grande domanda di cibo nel mondo. Le leggi che disciplinano le

\footnotetext{
${ }^{1}$ Laureato nel corso delle lettere presso l'Università Statale di Bahia - UNEB Campus X; Post laureato in portoghese dall'Università di Vale do Cricaré e uno studente regolare del master in educazione presso I'Università Internacional Unigrendal online.

${ }^{2}$ Laureato in lettere da Uneb, laureato in inglese da Unimes, laureato presso i Collegi Integrati di Jacarepagu (FIG), e studente regolare del master in educazione presso I'Università Internazionale Unigrendal online.
} 
risorse idriche sono sempre più regolate al fine di preservare le risorse idriche e l'intero ambiente. II Brasile ha dimostrato che è necessario preservare, per quanto resta da fare per ridurre al minimo gli impatti causati dall'uomo all'ambiente.

Parole chiave: acqua, scarsità, conservazione, sostenibilità.

\section{INTRODUZIONE}

L'umanità ha raggiunto, soprattutto in questo secolo, innumerevoli conquiste mai immaginate dalla scienza e dalla tecnologia. Tuttavia, il problema dell'acqua e il mantenimento della vita sul pianeta ha preso gran parte della popolazione mondiale a chiedersi cosa fare per garantire che tutti abbiano accesso all'acqua, ma non sempre ha una risposta, perché l'essere umano non è sempre o quasi mai essere $D$ Conto della necessità di preservare questo bene molto prezioso.

Questa preoccupazione in futuro non avviene per caso, dopo tutto, i dati sulla distribuzione, la riserva e la disponibilità di acqua sul pianeta dimostrano la necessità di cercare alternative per la conservazione, come si dice da VICTORINO:

È noto che quasi tutta la superficie del pianeta Terra è coperta dall'acqua: dagli oceani, dai fiumi e dai laghi, dai corsi d'acqua e dai Sangha. Acqua da calotte polari, acqua piovana, un sacco di acqua... Ma in realtà non tutto è blu (il colore che copre la terra - a causa dell'acqua -quando si vede dallo spazio), perché tutta l'acqua del pianeta (1,37 miliardi di km 3) è costituita fondamentalmente da due tipi: acqua salata dei mari e acqua dolce di fiumi, laghi e sottosuolo. Tuttavia, il $97 \%$ del totale è occupato da acqua salata, inadatto al consumo. Con il cosiddetto "ciclo dell'acqua" (processo di evaporazione e ritorno dell'acqua in superficie sotto forma di pioggia, neve, grandine o gelo), l'acqua si ricompone in diversi modi dando vita alle piante e generando diversi Biomm seguendo il corso naturale della vita. La natura è saggia e solo perché quando l'acqua fuoriesce nell'atmosfera sotto forma di evaporazione, il sudore viene compensato con le precipitazioni. Questa differenza tra il volume 
d'acqua che cade e il volume di acqua che evapora è di circa 45000 chilometri cubi all'anno - che, in teoria, l'essere umano potrebbe spendere. Solo il $20 \%$ di questo totale è disponibile. L'acqua utilizzabile è nei fiumi, laghi, acqua piovana e acqua sotterranea. Tuttavia, tutti insieme corrispondono solo all' $1 \%$ del volume di Acqua dolce (VICTORINO, 2007, p. 16).

Figura 1-chart-"distribuzione dell'acqua sulla Terra"

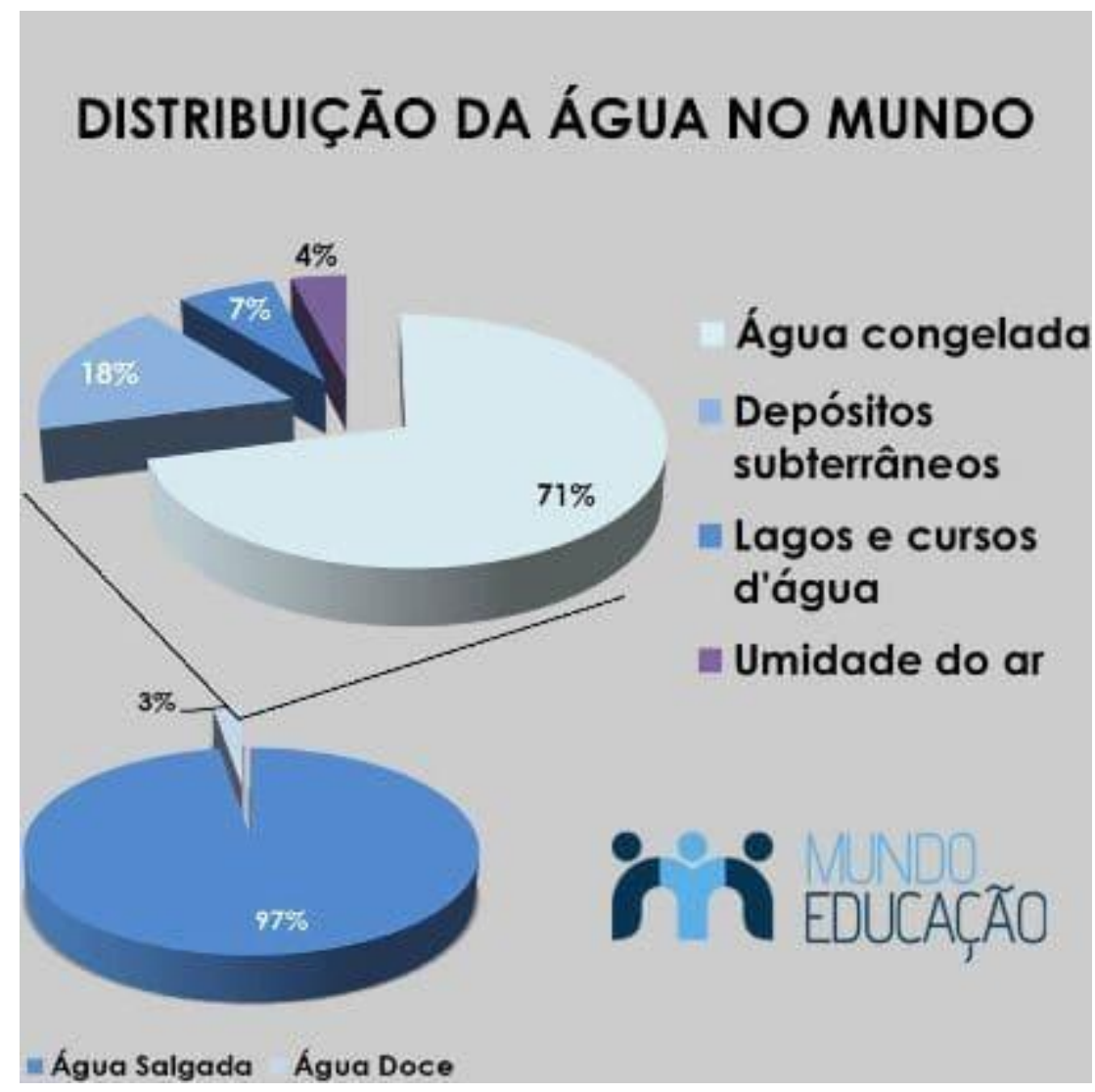

(Fonte: Sito Mondiale dell'Educazione)

Quando si analizza il grafico di cui sopra, si osserva che la quantità di acqua disponibile per l'uso è insufficiente, dal momento che:

Se raccogliamo 1,5 litri d'acqua, come la troviamo sul pianeta, e la dividiamo proporzionalmente, la quantità di acqua dolce disponibile equivarrebbe a una singola goccia insignificante. $\mathrm{E}$ a complicare, questo 
piccolo resto è sempre più inquinato, soprattutto nelle grandi città, a causa dell'aumento della popolazione mondiale che, solo nel secolo scorso è diventato tre volte maggiore, il che ha causato l'aumento delle fabbriche, più rifiuti e più irrigazione Nelle colture. Secondo la Banca mondiale, oggi circa 80 paesi si trovano ad affrontare problemi di approvvigionamento. La situazione più critica è in Asia, dove il $60 \%$ della popolazione vive con solo il $32 \%$ dell'acqua dolce disponibile (VICTORINO, p. 17).

Quando si analizzano tutti questi dati, è possibile valutare la gravità degli impatti causati dalle azioni dell'uomo all'ambiente $e$, in particolare, all'acqua, che è considerata una risorsa vitale per la vita sulla Terra. Molti paesi hanno già adottato posture di contenimento e conservazione attraverso trattati, accordi, al fine di ridurre al minimo tali impatti. Tuttavia, una parte considerevole della popolazione mondiale continua a ignorare che il futuro è minacciato e che ci sarà un cambiamento, non solo di mentalità, ma anche di atteggiamento verso l'uso di questa risorsa che è stata utilizzata in modo irresponsabile per lunga storia.

È noto che la popolazione mondiale è cresciuta in modo disordinato, e questo richiede anche un notevole aumento della produzione agricola industriale nel tentativo di garantire cibo a tutti, il che impone pressioni sempre più intense sull'ambiente. In considerazione di ciò, è necessario trovare strategie che cancellino gli impatti, dal momento che si prevede una popolazione di oltre otto miliardi di persone per l'anno 2020:65\% nelle zone costiere e il 60\% nelle città con più di 2,5 milioni di persone. Inoltre, l'uso dell'approvvigionamento idrico è aumentato enormemente in un breve periodo di tempo: mentre la ristrutturazione non ha cambiato il livello delle risorse idriche, dal 1900 al 1995, in contropartenza, la quantità utilizzata è aumentata a sei volte di più (il doppio aumento della popolazione) e due volte di più dal 1975. Dell'acqua totale consumata sul pianeta, l'agricoltura assorbe una media globale del $70 \%$ delle forniture idriche, e nei paesi sottosviluppati la percentuale è ancora più alta registrando un aumento dell' $80-90 \%$, con una media del $20 \%$ per l'industria e del $10 \%$ per gli usi domestici e OR Tros. 
Quando si analizza la situazione del Brasile in questo contesto, è ancora più evidente che l'acqua non è stata utilizzata in modo razionale, che compromette e molto l'offerta e la qualità della vita in molte regioni del paese.

\section{CONSUMO DI ACQUA NEL PAESE}

Figura 2-chart - consumo di acqua nel paese

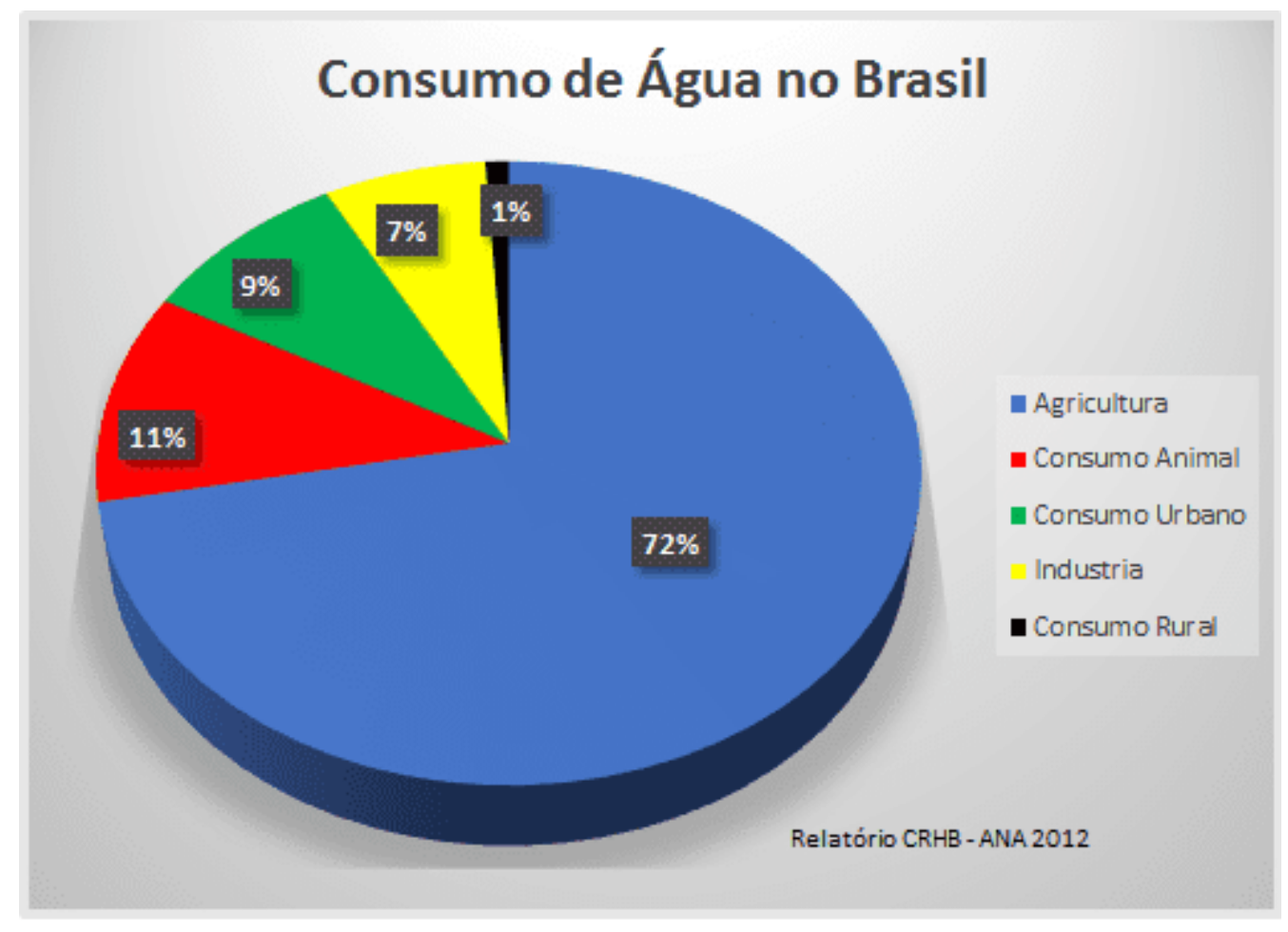

(Fonte: Consulenti EOS)

Il grafico di cui sopra indica l'agricoltura come il grande cattivo nel consumo e nello spreco di acqua in Brasile e, anche se il paese è un pioniere nell'approvazione di leggi che limitano il consumo e impone alcune sanzioni a coloro che commettono abusi nel consumo e nell'uso dell'acqua , è anche noto che non c'è supervisione insufficiente da parte dei governanti per garantire che le leggi siano rispettate, cioè, anche se ci sono leggi di conservazione, in pratica è ben lungi dall'accadere.

Al giorno d'oggi, nonostante il concetto giuridico di buon uso comune delle persone, l'acqua è una risorsa, per molti, di valore economico. Ciò è il risultato della crescente 
domanda di acqua per i vari usi, che accompagna lo sviluppo urbano e industriale dei paesi. Al giorno d'oggi, i grandi problemi legati all'acqua non si verificano a causa della natura, ma derivano da un uso improprio con rifiuti e improvident, nonché da un inquinamento diffuso che minaccia l'ambiente.

Il codice dell'acqua, la legge più grande della disciplina dell'uso dell'acqua in Brasile è il 10 luglio 1934, è già una certa età, ma non così non è più attuale perché classifica le categorie giuridiche, discrimina gli usi dell'acqua e dei suoi precetti e , soprattutto: cerca di garantire gli interessi generali della società. Nel vecchio codice è stato possibile trovare il dominio idrico privato in alcuni casi, ma questo è stato estinto dall'ottobre 1988, quando tutti i corpi $D$-acqua è diventato di dominio pubblico.

\section{LA QUALITÀ DELL'ACQUA CHE CONSUMIAMO}

La qualità dell'acqua disponibile sul pianeta è un altro aspetto che è stato anche oggetto di preoccupazione da parte delle agenzie sanitarie di tutto il mondo in quanto le malattie sono evitate quando si utilizza l'acqua decontaminata. La qualità dell'acqua ha deciso la fortuna di alcune regioni del mondo di carattere economico, soprattutto in relazione alla produzione di birre. Ma non è solo nel settore dei birrifici che la purezza dell'acqua è importante, è anche necessario nelle industrie degli arazzi, nella conceria, nell'industria del tessuto e nelle pietre preziose. Inoltre, la qualità dell'acqua determinerà la qualità della vita dei suoi consumatori. Quando non c'è acqua potabile adeguata per il consumo umano, il risultato è l'alto tasso di malattie e altri problemi che ne derivano. Questa è una preoccupazione che in passato ha determinato il successo o il fallimento delle nazioni più sviluppate, come dice Célia Jurema:

L'uomo si preoccupava sempre dell'acqua. Per 4.000 anni a.C., le prime leggi che erano note erano codici che regolavano l'uso dell'acqua, scritti dai Sumeri. Ma non tutte le civiltà sono state attente a questo proposito. I documenti mostrano che i Maya hanno dovuto abbandonare la città di Tical, situata in una foresta tropicale piena, dove sono state trovate le rovine della Piramide del Sole, perché non sapevano come immagazzinare correttamente l'acqua, oltre a produrre crescenti erosioni 
e grandi Deforgia perché usavano il legno anche nelle strutture interne delle colonne dei loro giganteschi templi. In Messico, nella penisola dello Yucatan, il dio della pioggia, Chac, era venerato e, ogni volta che l'acqua diventava scarsa, venivano eretti nuovi templi nella speranza che il dio delle acque salvasse i Maya dalla siccità. Si trovano spesso, motivi del Dio pioggia Maya alla base delle piramidi. La coesistenza con i fiumi ha portato saggezza alle popolazioni durante lo sviluppo dell'umanità, i faraoni, hanno pianificato le loro città lasciando le aree lungo il fiume Libero "(VICTORINO, 2007, p. 19).

Fin dai tempi più remoti, l'acqua è sempre stata una delle più importanti autorità di regolamentazione sociale. Le strutture delle società contadine e delle comunità dei villaggi, dove le condizioni di vita sono intimamente legate al suolo, sono state organizzate intorno all'acqua. Ci sono stati rari casi in cui tutti i membri di una comunità erano allo stesso livello in relazione all'acqua - l'accesso ad essa quasi sempre comportava disuguaglianze. Questo ci porta a capire come sia il rapporto degli esseri umani con l'acqua e gli uni con gli altri a causa dell'acqua. L'uomo, tuttavia, ha impiegato molto tempo per prendersi cura e curare efficacemente la qualità dell'acqua - molti ancora oggi non si sono resi conto che, più giorni meno giorno, la scarsità sarà una realtà, e continuare a utilizzare l'acqua indispenalmente è per compiti semplicistici Es come lavare l'auto con tubo o spazzare il marciapiede con Jets D - acqua. Altri ancora suonano tutti i tipi di rifiuti in laghi, fiumi e torrenti. Per quanto tempo la società continuerà ad agire in modo irrilevante e irresponsabile? L'acqua, questo minerale che entrambi conosciamo e che fa parte di noi, esiste da almeno 3,9 miliardi di anni. II "essere razionale", in poche generazioni, è già riuscito a scendere a compromessi, non solo la qualità, ma anche la quantità di questa primavera naturale. Si può valutare il grado di sviluppo di un popolo dalla qualità dei servizi idrici e igienico-sanitari che vengono offerti alla popolazione. La capacità di sostenere la vita e la società umana è complessa, dinamica e varia a seconda di come l'uomo gestisce le risorse ambientali. È strano che i saggi sacerdoti dell'antichità non abbiano percepito le correlazioni esistenti tra l'approvvigionamento idrico e la conservazione delle foreste. A quel tempo, 
piantare era il privilegio solo dei principi, non era attraverso le loro ' lucide ' teste che richiedevano fronti di fioriere per riforestare le terre ravite:

8000 anni fa, il Brasile aveva il 9,8\% delle foreste del mondo. All'inizio del XXI ST, questa percentuale è scesa al 28\% e, peggio ancora, i 64 milioni di chilometri quadrati delle foreste esistenti prima dell'espansione demografica e tecnologica degli esseri umani rimangono meno di 15,5 milioni, circa il $24 \%$. Ciò significa che più del $75 \%$ delle foreste del mondo sono già scomparse... Ebc

Ripristinare le risorse naturali degradate è più difficile della sua conservazione; $E$, molti di loro, come l'acqua, non possono essere ripristinati, quando finisce non si può tornare indietro, quando finisce è per sempre. La falsa illusione che l'acqua non finisca mai evidente in natura giustifica forse, in parte, l'incuria storica degli esseri umani nei loro rapporti con le risorse idriche. È noto che non c'è così tanta acqua potabile disponibile come il paesaggio ci fa vedere. Quello che abbiamo come acqua potabile è solo lo $0,03 \%$ dell'acqua totale del pianeta. Questa quantità insignificante dovrebbe ricevere tutte le cure possibili, tuttavia, questo non è ciò che vediamo in quasi tutti i continenti, dopo tutto, le principali falde acquifere si stanno esaurendo con una velocità superiore alla loro naturale capacità di recupero.

\section{L'USO INDISCRIMINATO DELL'ACQUA E L'IMPEGNO DELLA QUALITÀ DELLA VITA DELL'UMANITÀ}

Non è necessario andare troppo lontano per percepire situazioni di spreco e svalutazione delle risorse naturali. Con l'acqua è ancora la stessa, e in teoria l'idea di preservare questa risorsa pur essendo in qualche modo enfatizzata, in pratica non c'è grande mobilitazione per prevenire conseguenze prevedibili di un futuro con scarsità d'acqua. La seguente citazione definisce chiaramente la situazione in questione:

Una delle principali sfide della società in questo $X X I$ secolo riguarda l'urgente necessità di conservare le risorse idriche in termini di qualità e 
quantità, in uno scenario di aumento della popolazione e bassa sostenibilità dei processi produttivi e dei consumi.

[...]Queste previsioni di crescita della popolazione e le stime legate alla produzione alimentare, alla conservazione e alla distribuzione considerano che se la popolazione mondiale raggiungerà i 10 miliardi di abitanti nei prossimi 50 anni, avremo il 70\% degli abitanti del pianeta Affrontare le carenze nell'approvvigionamento idrico, colpendo circa 1,06 miliardi di persone che non avranno nemmeno acqua per il cibo di base (SILVA, 2009, p. 1).

Mentre in molti paesi del mondo l'acqua è già diventata una disputa che genera conflitti diversi, il Brasile non ha ancora realizzato la grande responsabilità che abbiamo con la qualità della vita delle generazioni future. Secondo Célia Jurema Aito, il Brasile è un paese privilegiato in termini di disponibilità di acqua in quanto conta con il $28 \%$ della disponibilità sudamericana e il $12 \%$ delle riserve idriche mondiali. In territorio brasiliano, il 72\% dell'acqua si trova nel bacino amazzonico. II Rio delle Amazzoni ha 6.885 chilometri di estensione ed è il più grande volume d'acqua al mondo, scaricando 175 milioni di litri al secondo nell'Oceano Atlantico. Tuttavia, non si può dimenticare che anche la crescita della popolazione comporta il rischio di scarsità. Secondo Célia Jurema, tra il 1970 e il 2000, il Brasile è passato da una popolazione urbana del 55\% all' $82 \%$ della popolazione totale. È noto che più di 1,4 miliardi di persone non hanno accesso all'acqua potabile e altri 2 miliardi non hanno alcun tipo di servizi igienicosanitari di base.

Come l'uomo moderno si è evoluto in tecnologia, ha cessato di avere contatto con la sua essenza naturale, ha continuato a vivere sempre più lontano dalla sua base, la natura, e per diventare un essere ansioso e irritato. Lo squilibrio collettivo che il nostro pianeta deve affrontare rappresenta profondamente uno squilibrio degli individui che compongono la popolazione mondiale. Questo squilibrio si riferisce a un fallimento nel processo di evoluzione dell'uomo che si distoglie dalle sue origini, cerca, la maggior parte delle volte, il suo benessere individuale, dimenticando che siamo parte di un organismo vivente chiamato "società" e per farlo Sviluppare in modo efficace, è 
necessario garantire il bene di tutti e non solo di se stessi. Nelle grandi città, si percepisce che sempre più persone si sentono sole, indifese, persino vivendo in mezzo alla folla, il che le rende ogni giorno più individualistiche ed egoiste. $E$ anche se al giorno d'oggi, c'è un'idea di conservazione, almeno nella pubblicità di molte aziende che mirano a sollevare la bandiera della conservazione di un "business marketing" che è redditizio, la grande verità è che poco fare per la conservazione dell'ambiente e Di conseguenza l'acqua nel paese. Pertanto, riteniamo che l'inefficienza, nonostante tanti sforzi, dei nostri governanti, delle organizzazioni non governative e persino della popolazione stessa in relazione alla questione ambientale, poiché tutto il lavoro è fatto fondamentalmente per risolvere i problemi Quando il processo di cambiamento e miglioramento dovrebbe iniziare al livello interno di ogni persona.

Purtroppo, la popolazione mondiale che occupa sempre più spazio sul pianeta è direttamente responsabile del degrado dei fiumi, delle zone costiere, degli oceani, delle spiagge e, poiché questa popolazione non cresce, il volume dei rifiuti è diventato ogni giorno più grande. Per avere un'idea del problema, solo gli americani producono 212 milioni di tonnellate di spazzatura all'anno e spostano quasi 3 milioni di bottiglie di plastica all'ora. Ci vuole una coscienza per smaltire questo tipo di rifiuti in luoghi di riciclaggio. In Brasile, la politica nazionale dei rifiuti solidi (legge federale 12.305/2010) determina che tutto il materiale prodotto dalle attività domestiche e commerciali che sarà possibile raccogliere dai servizi di pulizia pubblici, deve essere inoltrato alla destinazione Terminare solo quando non è possibile riutilizzarlo, sia attraverso il riciclaggio, il riutilizzo, il compostaggio o la generazione di energia. Laddove non esiste una tecnologia praticabile, i rifiuti devono essere destinati alle discariche. Tuttavia, del volume prodotto nel 2016, quasi 30 milioni di tonnellate non hanno la destinazione appropriata, che rappresenta una percentuale del $41,6 \%$ del totale generato, che trasforma i rifiuti solidi in un grave problema ambientale, perché uno smaltimento inadeguato può Caricare questo materiale in corsi d'acqua e fiumi e quindi raggiunge gli oceani, oltre al problema delle discariche, l'impatto sulla salute pubblica tra gli altri effetti negativi. 
In media vengono prodotti 80 milioni di tonnellate di rifiuti solidi urbani, essendo quasi 470 chili per abitante, dove il $60 \%$ di questo totale è materiale riciclabile. Tuttavia, solo il $3 \%$ dei rifiuti prodotti viene inoltrato per il riciclaggio.

L'impressione è che ovunque le acque superficiali siano inquinate da una spaventosa varietà di rifiuti urbani, industriali e agricoli. Anche nei paesi industrializzati, dove è in vigore una legislazione sulla qualità dell'acqua, l'inquinamento è ancora un problema che preoccupa. Negli Stati Uniti, si è scoperto che il $60 \%$ dei laghi intervistati sono inquinati da sostanze nutritive provenienti da fertilizzanti e letame animale, che sta causando una morte quasi massiccia dei pesci, oltre al degrado della qualità dell'acqua. In Canada, 20000 laghi sono colpiti da piogge acide.

La crescita demografica ed economica del Brasile negli ultimi anni ha utilizzato le risorse idriche ben oltre la sua capacità di sostenere sia la quantità che la qualità. I gravi problemi di gestione dell'acqua sono causati dall'espansione disordinata delle popolazioni urbane e dalla limitata disponibilità di risorse idriche, inoltre, le politiche pubbliche volte a preservare le acque sono insufficienti di fronte Domanda di consumo e utilizzo. L'occupazione degli spazi pianeggianti, vicino ai fiumi e senza controllo, ha causato per anni e anni, piccole inondazioni in un gran numero di città nel sud e sudest, in Brasile. Negli anni 80 si sono verificate diverse inondazioni lungo il fiume e con perdite importanti, principalmente a Paranà, Santa Catarina, San Paolo e Minas Gerais. Nel nostro paese c'è una grande diversità di situazioni, con molte risorse idriche nelle regioni settentrionali e del Midwest e scarsità nelle regioni nord-est e sudest, in opposizione alla maggiore concentrazione della domanda, insieme alla regione meridionale. Lo sviluppo urbano aumenta anche le aree resistenti all'acqua e a basso assorbimento attraverso residenze, passeggiate, strade, parcheggi e persino parchi. Poiché l'acqua piovana non può infiltrarsi nel suolo, il volume aggiuntivo si riversa nel sistema di drenaggio verso i fiumi. Nel decennio 70, c'era grande speranza che fosse possibile pianificare la crescita delle città e pianificare il loro futuro armonicamente. Tuttavia, 37 anni dopo, sembra che l'urbanistica in Brasile, o anche all'estero, sia stata atrofizzata portando all'accumulo di problemi. 
Il lungomare brasiliano è vittima di furia immobiliare, soprattutto a Rio de Janeiro, oltre alle spiagge del nord-est e del sud. La crescita rampicante fornisce l'emergere di villaggi e favelas dove l'acqua potabile non si trova e, molto meno, le acque reflue. Rio de Janeiro e San Paolo, le due maggiori città brasiliane, hanno gravi deficit di rete di trattamento delle acque reflue e, pur avendo prestiti internazionali, non riescono a ridurre l'alto livello di inquinamento della baia di Guanabara e del fiume Tiet A causa della mancanza di consapevolezza della popolazione e di un programma di educazione ambientale che stimola i cambiamenti nel comportamento degli individui.

Le più grandi città del paese gestiscono male le loro risorse idriche. Nel 1997, la Banca Mondiale ha offerto 40 miliardi di dollari per le società di servizi igienico-sanitari. Quasi il $100 \%$ del denaro rimane ancora intatto perché le società brasiliane non rientrano nei requisiti tecnici minimi per ricevere prestiti-affermazione del geologo Aldo Rebouas, della società di ricerca sulle risorse minerarie - questo perché la banca Determina che le aziende hanno una perdita massima del $20 \%$ nelle reti di distribuzione idrica. Solo a San Paolo, nel 2001, le perdite sono state del $30 \%$.

Il degrado ambientale è uno degli aspetti più critici del processo di deterioramento causato direttamente e indirettamente dall'uomo. Le regioni che in precedenza avevano quantità di risorse idriche, oggi cominciano a dare segni di scarsità, e la spiegazione è lo spreco con uno sfruttamento eccessivo, il limo dei fiumi e l'inquinamento delle fonti. E tutti questi problemi nascono quasi sempre dall'esplosione dell'agricoltura industriale che serve a sfamare i milioni di abitanti delle città. È un dato di fatto che l'urbanizzazione e la crescita dilagante delle città aumentano significativamente il consumo e l'uso improprio dell'acqua.

\section{COSA FARE DI FRONTE A TALI RIFIUTI?}

La disidratazione dell'acqua non è solo un problema tecnico, ma anche di ordine economico e sociale, perché non ci sono risorse sufficienti per impiantare i sistemi di depurazione delle acque reflue liquide con la tecnologia disponibile. È noto che gli Stati sono stati in grado di acquisire risorse attraverso banche internazionali e fare i primi passi a favore delle nostre fonti, come a Rio Grande do Sul, con il Prà-Guaba 
(programma statale) e Guaaba Vive, nella capitale Porto Alegre, come Riferimento al problema delle risorse idriche. Tuttavia, c'è ancora molto da fare, perché l'acqua è necessaria in tutti gli aspetti della vita. Le risorse d'acqua dolce costituiscono una componente essenziale dell'idrosfera terrestre e una parte indispensabile di tutti gli ecosistemi terrestri, e la sua diffusa scarsità, la distruzione graduale e l'aumento dell'inquinamento delle fonti idriche in molte regioni del mondo La consapevolezza della domanda e i cambiamenti negli atteggiamenti nei confronti dell'acqua. E mentre molti squandere e rifiuti, altri già soffrono le conseguenze della scarsità d'acqua. La disuguaglianza e i rifiuti di distribuzione sono due ragioni forti che spiegano in parte il motivo per cui 1,4 miliardi di persone - quasi cinque volte la popolazione degli Stati Uniti - non hanno accesso all'acqua potabile, e l'acqua è un fattore primario per la salute. È anche noto che il $60 \%$ delle risorse idriche si trova in soli nove paesi, tra cui il Brasile, ma sono 80 paesi che rappresentano il $40 \%$ della popolazione mondiale, che soffre di scarsità d'acqua. Tuttavia, ciò non significa che gli Stati Uniti, il Brasile, la Russia, il Sudafrica o la Cina, considerati paesi ricchi di acqua, non siano esenti da problemi di mancanza di acqua.

Per quanto riguarda i rifiuti, secondo Rodolfo F. Alves Pena, l'agricoltura è responsabile dell'assorbimento medio mondiale, del $70 \%$ delle forniture idriche, e questo indice va all' $80-90 \%$ nei paesi sottosviluppati. L'acqua potabile viene persa in media del $50 \%$ nelle perdite dei sistemi di distribuzione e se non si fa nulla nel senso di cambiamenti comportamentali, nell'anno 2025 saranno più di 4 miliardi di persone che non avranno accesso a questo preziosissimo ed essenziale per la vita.

Pertanto, è necessario analizzare la questione dei rifiuti d'acqua nell'ambiente rurale in modo integrato, in cui diversi fattori influenzano il cambiamento nell'abitudine dei produttori, nel caso dell'uso corretto delle risorse idriche, come si discute Oliveira (2008):

La decisione e l'opzione per un uso più efficiente delle risorse naturali in generale e dell'acqua in particolare dovrebbero essere fornite in modo integrato e associate a strumenti di gestione a tutti i livelli pertinenti. Solo in questo modo sarà possibile raggiungere un livello che consenta 
un'adeguatezza tra domanda e offerta in vista dell'uso razionale di una risorsa così preziosa. (OLIVEIRA, 2008, p.3)

Questa consapevolezza deve avvenire nei vari settori della società, indipendentemente dalla condizione sociale, culturale e intellettuale degli individui. Questo perché oggi un cittadino europeo consuma 150 litri di acqua al giorno, mentre un indiano deve accontentarsi di soli 25 litri al giorno. Un americano consuma 3.000 litri di acqua al giorno.

E 'bene sottolineare che lo spreco della carta è più un aggravante nel abbattuto degli alberi, così come il consumo di acqua, dal momento che, per produrre una tonnellata di fibra vergine, è necessario 44000 a 83000 litri di acqua. È necessario incoraggiare l'uso di carta riciclata, poiché, per ogni tonnellata di carta utilizzata, si può produrre quasi una tonnellata di carta nuova (più efficiente del rapporto di 2-3,5 tonnellate di alberi per produrre una tonnellata di carta vergine). II processo di educazione ambientale dovrebbe essere stimolato.

L'acqua dolce, una risorsa limitata, è altamente vulnerabile e di molteplici usi, quindi deve essere gestita in modo integrato con meccanismi di coordinamento e attuazione efficaci. Nei piani per il suo utilizzo, è necessario sottolineare la protezione, la conservazione e la gestione sostenibile e razionale in base alle esigenze e alle priorità di ogni comunità. La valutazione delle risorse idriche è la base pratica per la sua gestione integrata e sostenibile, nonché un esame equilibrato delle esigenze della popolazione e dell'ambiente.

Per tutte le ragioni qui presentate, è necessario tenere a mente che l'acqua è il bene più prezioso di questo secolo, questo, tuttavia, non significa che si debba trasformare la "fonte di vita" in un sistema monetario, generatore di tanti conflitti, ma piuttosto unire gli sforzi a favore del consumo consapevole e la conservazione di questa risorsa vitale per garantire la qualità della vita delle generazioni future. 


\section{CONSIDERAZIONI FINALI}

La sostenibilità è il rapporto tra i sistemi dell'economia umana e l'ambiente. Le azioni degli uomini devono essere in sintonia con l'ambiente, garantendo manutenzione e conservazione per mantenere la diversità, la complessità e il funzionamento del sistema ecologico che ci tiene in vita. $E$ pensare in modo sostenibile è necessario, perché le risorse naturali sono limitate. Inoltre, la qualità delle risorse è diventata sempre più peggiore a causa dell'aumento della popolazione e della mancanza di politiche pubbliche volte a preservarle. Ad esempio, si stima che circa 12 milioni di persone muoiano ogni anno a causa di problemi legati alla qualità dell'acqua.

L'unico modo in cui la maggior parte dei paesi può trattare il crescente inquinamento delle loro risorse idriche è l'attuazione di strategie di gestione integrate, che non solo aiutano a pulire i corsi D - Acqua, ma in primo luogo, anche prevenire l'inquinamento. Finora, pochissimi paesi sono stati effettivamente in grado di praticare tali cambiamenti. Devi stare attento. Dovremo evitare sprechi, interrompere i processi inquinanti e creare nuovi modi per controllare, catturare e distribuire.

Ci vuole una risposta culturale ed etica. La situazione con cui passa il mondo, non è altro che uno stile di vita più sofisticato, in cui ciò che si vuole "must have", causando così l'accelerazione delle fabbriche e delle industrie che sono tra i maggiori utilizzatori di acqua solo perdendo a causa dell'agricoltura e dell'uso del poliestere plastica. Nel XX secolo la popolazione è cresciuta tre volte, mentre il consumo di acqua è aumentato di sei. Anche noi brasiliani, che hanno il 16\% di tutta l'acqua del mondo, soffrono di razionamenti dovuti alla cattiva amministrazione delle risorse e all'inadeguatezza delle infrastrutture. I paesi aridi si trovano ad affrontare sfide più spaventose come il Medio Oriente, dove ci sono aree in cui la scarsità limita la crescita socio-economica.

Le misure di gestione congiunte per l'uso delle risorse idriche e terrestri dovrebbero essere più frequenti al fine di aumentare l'efficienza dell'uso dell'acqua di irrigazione, evitando così l'erosione del suolo, le inondazioni, i sedimenti, la salinizzazione e gli effetti nocivi Pesticidi e fertilizzanti negli organismi. Inoltre, il degrado ambientale ha 
generato una patologia ambientale emergente e malattie come il colera e la dengue sono diventate più fortemente causate dalla contaminazione dell'aria, dell'acqua e dei suoli, nonché dall'uso di sostanze tossiche e pericolose, come Pesticidi e altri prodotti per la cura speciale. Come abbiamo visto finora, il degrado non è solo dell'ambiente, ma anche dell'essere umano, i cui valori vanno perduti, dove le necessità non sono più le basi, e piuttosto il superfluo, dove sbagliare è quello giusto. È qui che vedremo l'educazione ambientale come una priorità per raggiungere gli obiettivi dello sviluppo sostenibile. La conferenza di Tbilisi tenutasi nel 1977, che si occupa di educazione ambientale a livello internazionale, rende il vedere l'EA come la formazione di una coscienza fondata su una nuova etica che resisterà a ogni sfruttamento. Questo è ciò di cui l'umanità ha bisogno, per prendere una svolta nelle sue azioni, nei suoi investimenti, nei suoi atteggiamenti, nei suoi valori, per rendersi conto che non solo sta pensando a se stessa che sarà in grado di dare un futuro sano alle sue generazioni.

Sappiamo che l'uomo è il principale chiazza di così tanto squilibrio. L'uomo ha disturbato così completamente l'ambiente naturale in cui si è trasferito che non conosce più il suo posto in questo sistema, tranne, naturalmente, come fattore di disordine. Finora i nostri tentativi di dominare il mezzo consistevano in semplici riforme del paesaggio, condotte maldestrae e irriflesamente; Mentre altre attività umane hanno prodotto dannosi, imprevisti e mal compresi effetti collaterali. Tutti gli oceani sono stati inquinati dagli esseri umani, dall'atmosfera e persino dagli strati glaciali remoti. La maggior parte dei fiumi sono più o meno inquinati e molti di loro, come il fiume Tietà $\mathrm{e}$ il Tevere, sono stati trasformati in acque reflue ad aria aperta. Barbie Nadeau, Giornalista di Newsweek nel 2002 era a Roma e dà la sua testimonianza sul Tevere "... Galleggiano pesci morti decimati, non conosciuti per cosa. II fetore è ovunque, un residuo oleoso rende le anguille e le carpe morte bagliore "e conclude:"... Se un fiume è la linea simbolica della vita di una città, allora Roma è in una situazione piuttosto brutta. " II caso non è quello di dominare il mezzo, ma di sapere se la natura può essere preservata con un certo aspetto dell'ordine e se la civiltà è in grado di sopravvivere alla trasformazione che provoca in natura. L'evoluzione culturale $\mathrm{ci}$ ha gradualmente portato a riconoscere che l'umanizzazione del pianeta può avere successo solo se vengono rispettate le leggi ecologiche fondamentali. 
È ora di iniziare a pensare di cambiare abitudini. Sentire che formiamo insieme un'unica catena; Se si rompe un collegamento, la catena non avrà più senso. Tutti hanno bisogno di tutti. Gli studiosi indiani, migliaia di anni fa, hanno già detto che il tutto è contenuto nelle parti e, per quanto le cose sembrano diversificate e plurali, infatti, sono aspetti della stessa e unica realtà. Ecco una frase da mediare, da tutti noi, in modo che possiamo capire le dinamiche della vita e vedere che non c'è me e sì, i nodi. Finora la natura è stata utilizzata gratuitamente, e grazie ad essa produciamo e viviamo. Tuttavia, nel panorama della crescita della popolazione mondiale non ha riconosciuto il suo valore dovuto.

Per quanto riguarda l'irrigazione, responsabile dei rifiuti e del conseguente impegno delle sorgenti, per ridurre al minimo l'impatto del suo uso inadeguato, Rebou (2001) sottolinea che è necessaria una pianificazione più efficiente e razionale dell'uso dell'irrigazione e dell'ottimizzazione dell'attrezzatura utilizzata. Può anche ridurre i costi dell'elettricità.

Un'altra pratica recente in Brasile e che si è rafforzata è l'uso dell'acqua di riutilizzo per soddisfare la necessità di irrigazione di diverse colture alimentari. Hespanhol (2002) integra che in diversi paesi in cui viene utilizzata questa tecnica, la produttività aumenta in modo significativo quando ben pianificata.

Qualsiasi forma di uso efficiente dell'acqua finalizzata a ridurre al minimo i rifiuti sul campo dipende direttamente dalla pianificazione effettuata dal produttore. La pianificazione è indispensabile per armonizzare i vari usi dell'acqua, consentendo ai diversi settori produttivi, monitorando la quantità e la qualità delle risorse idriche, migliorando i livelli di efficienza complessiva dell'uso (PA, 2000).

In secondo luogo (FURRIELA,2001) La prima grande sfida che si pone non solo per il produttore rurale, ma anche per tutta l'umanità è la consapevolezza che non vi è alcuna fornitura inesauribile di acqua potabile sul pianeta. Alcune misure dovrebbero essere promosse per garantire l'approvvigionamento di acqua in quantità e standard accettabili. La grande sfida di oggi è ridurre il livello di degrado dell'acqua mediante misure quali la conservazione, il miglioramento dei servizi igienico-sanitari di base, la 
riduzione dell'uso dei pesticidi, una più pulita gestione della produzione industriale e dei consumi.

Ogni giorno è più evidente la necessità di percepire l'acqua come un bene economico, in cui la sua gestione dovrebbe basarsi su principi di efficienza economica, in modo da soddisfacire la domanda da una prospettiva di sostenibilità.

Essa aggiunge inoltre che è necessario stabilire un nuovo modo di pensare e agire, adottando abitudini, usi e costumi diversi, dove l'obiettivo generale è la crescita economica, basata sul consumo sostenibile di acqua, promuovendo così la protezione delle Le sorgenti ancora conservate e il recupero di coloro che sono già stati danneggiati, egli sottolinea che questo non è sufficiente, ed è necessario fare molto di più per raggiungere questo obiettivo dovrebbe quindi trattare l'acqua come una priorità sociale e ambientale, poiché è necessario Prendersi cura delle risorse idriche brasiliane, poiché la crescente necessità di acqua mondiale porterà al paese la possibilità di sfruttare la sua crescita e lo sviluppo, e di mantenere un input essenziale per la vita e qualsiasi attività produttiva.

\section{RIFERIMENTI BIBLIOGRAFICI}

AGENDA 21. The Earth Summit Strategy to save our Planet. Ed. Daniel Sitarz, Earthpress, 321p. Boulder Colorado, 1994.

AGÊNCIA NACIONAL DAS ÁGUAS. Disponível em: <www.ana.gov.br> Acesso em: $20 / 07 / 2019$

Baía de todas as águas - Preservação e Gerenciamento Ambiental na Bacia Hidrográfica do Guaíba - Pró-Guaíba, RS/1998.

EBC. Disponível em: <www.ebc.com.br/.../mais-de-75-das-florestas-primarias-domundo-nao-existem-mais >Acesso em:19/07/2019 
FURRIELA, R. B. EDUCAÇÃO PARA O CONSUMO SUSTENTÁVEL. Ciclo de Palestras sobre Meio Ambiente - Programa Conheça a Educação do Cibec/InepMEC/SEF/COEA, 2001.

HESPANHOL, I. Potencial de Reuso da Água No Brasil, Agricultura, Industria, Municípios, Recarga de Aquíferos. RBRH- Revista Brasileira Dos Recursos Hídricos. Vol. 7 n. 4, 2002

JUSBRASIL. Disponível em: <https://www.jusbrasil.com.br/topicos/10603350/artigo90-do-decreto-n-24643-de-10-de-julho-de-1934> Acesso em 20/07/2019

MUNDO DA EDUCAÇÃO. Disponível em: <https://mundoeducacao.bol.uol.com.br/geografia/consumo-agua-no-mundo.htm> Acesso em 12 de setembro de 2018.

OliveiRA, E. C. A Gestão Dos Recursos Hídricos Sob a Perspectiva da Descentralização. ANAP BRASIL - REVISTA CIENTÍFICA, ano 1 no 1, 2008.

PORTAL FMU. Disponível em: <https://portal.fmu.br/reciclagem-no-brasil-panoramaatual-e-desafios-para-o-futuro/> Acesso em: 20/07/2019

PUC RS. Disponível em: $<w w w . p u c r s . b r / e d i p u c r s / o n l i n e / p l a n e t a a g u a / p l a n e t a a g u a . p d f>$ Acesso em: $20 / 07 / 2019$

REVISTA EDUCAÇÃO AMBIENTAL EM AÇÃO. Disponível em: $<$ http://www.revistaea.org/artigo.php?idartigo=1271 > Acesso em: 17/07/2019

REBOUÇAS, A. C. Água e desenvolvimento rural. ESTUDOS AVANÇADOS, 2001.

REBOUÇAS, A.C. Água no Brasil: Abundância, Desperdício e Escassez.BAHIASalvador, v. 13, n. ESPECIAL, p. 341-345, 2003 ANÁLISE \& DADOS

SILVA,J. S. Água e Agricultura Irrigada. Palestra-Seminário Agricultura Irrigada Com Desenvolvimento Sustentável-Universidade Federal De Uberaba-Em 24-09-2009. 
http:www.irrigacao.org.brdocdownloadAgua\%20Agricultura\%20Irrigada_JoseSilverio. pdf.. Acessado em: 20/07/2019

TUCCI, C. E. M. Gestão da água no Brasil. Brasília: UNESCO, 2001. 156p.(1. Hidrologia - Brasil II. Hespanhol, Ivanildo III. Cordeiro Netto,

VICTORINO, C. J. A. Planeta água morrendo de sede: uma visão analítica na metodologia do uso e abuso dos recursos hídricos. Porto Alegre: EDIPUCRS, 2007.231 p. (1. Água - Uso. 2. Água - Qualidade. 3. Recursos).

Inviato: giugno 2019.

Approvato: agosto 2019. 